\title{
Alteridad de la educación para impulsar y valorar la sustentabilidad
}

\author{
Pedro César Cantú-Martínez* \\ Universidad Autónoma de Nuevo León, Nuevo León, México \\ https://orcid.org/0000-0001-8924-5343
}

Recibido: 21/08/2019

Revisado: 15/12/2019

Aceptado: 05/05/2020

\begin{abstract}
Resumen
La presente revisión teórica se sustenta en el reconocimiento de la necesidad de plantear un nuevo tipo de desarrollo que garantice la continuidad del ser humano y de todo cuanto le rodea. Por lo tanto, al acoger la sustentabilidad como concepto integrador de los aspectos económicos, sociales y ambientales, se pueden construir valores y actitudes que permitan lograr nuevos modos de vida. En ese sentido, la educación se presenta como el instrumento social que nos permite orientar la conducta humana a través de la transmisión del conocimiento. Nuestro objetivo ha sido internalizar con esta temática y destacar el papel que tiene la educación, como las instituciones educativas, para promover la sustentabilidad desde el marco de los Objetivos del Desarrollo Sustentable. Para ello se estableció un marco metodológico empírico analítico para destacar que la educación se erige como un proceso de humanización que nos conduce a una nueva visión del mundo y del propósito de vida. De tal manera, las instituciones de educación tienen un papel relevante: resignificar los imaginarios simbólicos y sortear las visiones que deterioran el ambiente y al ser humano en la tarea de construir una ciudadanía alfabetizada a partir de la sustentabilidad.
\end{abstract}

Palabras Clave: educación, sustentabilidad, valores, desarrollo sustentable.

\section{A shift in education to instill and value sustainability}

\begin{abstract}
This theoretical review highlights the need to advocate a new type of development in order to ensure the continuity and harmony between human beings and their environment. This is why sustainability, as a concept that integrates economic, social and environmental aspects, will lead us to build values and attitudes that generate new lifestyles. Education is thus regarded as a social instrument that allows providing guidance for human behavior through the transmission of knowledge. Our objective was to become familiar with the topic at hand and highlight the role it plays in education, as well as educational institutions, so as to promote sustainability within the framework of the Sustainable Development Goals. In order to do so, we used an empirical-analytical methodological approach to outline that education is put forth as a humanization process that offers a new vision of the world and meaning of life. Therefore, educational institutions play an important role in providing a new meaning to symbolic and imaginary constructs and circumventing visions that are detrimental to the environment and ensuingly to human beings whose aim is to develop an environmentally literate citizenry through sustainability.
\end{abstract}

Keywords: Education, Sustainability, Values, Sustainable Development. 


\title{
Mudança na educação para promover e valorizar a sustentabilidade
}

\begin{abstract}
Resumo
Esta revisão teórica sustenta-se no reconhecimento da necessidade de propor um novo tipo de desenvolvimento que garante a continuidade do ser humano e de tudo aquilo que o rodeia. Portanto, ao se aderir à sustentabilidade como conceito integrador dos aspetos econômicos, sociais e ambientais, é possível construir valores e atitudes que propiciem novas maneiras de vida. Neste sentido, a educação erige-se como o instrumento social que permite orientar a conduta humana através da transmissão do conhecimento. Nosso objetivo com esta temática tem sido internalizar e destacar o papel que a educação e as instituições educativas têm para promover a sustentabilidade desde o contexto dos Objetivos do Desenvolvimento Sustentável. Para isso, estabeleceu-se um quadro metodológico empírico analítico para destacar que a educação se erige como um processo de humanização que nos conduz a uma nova visão do mundo e do propósito de vida. De tal maneira, as instituições de educação têm um papel relevante: dotar os imaginários simbólicos de um novo significado e eludir as visões que deterioram o ambiente e o ser humano na tarefa de construir uma cidadania alfabetizada a partir da sustentabilidade.
\end{abstract}

Palavras-chave: educação, sustentabilidade, valores, desenvolvimento sustentável.

Citar como:

Cantú-Martínez, P.C. (2020). Alteridad de la educación para impulsar y valorar la sustentabilidad. Revista Digital de Investigación en Docencia Universitaria, 14(1), e1023. https://doi.org/10.19083/ridu.2020.1023

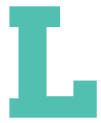
a revolución industrial impulsó un modelo socioeconómico que trajo consigo una visión de orden materialista donde se sujeta a la naturaleza y a un sector social de la comunidad humana al servicio de intereses y enriquecimientos particulares. Desde esta perspectiva, se reconoce lo que Beck (1998) denominó sociedad del riesgo. La cual se identifica por el menoscabo de las condiciones de vida, principalmente de las clases sociales menos favorecidas, y también de un progresivo deterioro ambiental que obtiene como resultado una fuerte crítica a las estructuras sociales que sostienen dicho modelo.

En este sentido, los retos que se han presentado y que como sociedad se han tenido que sortear, ha requerido de enfoques integradores de los ámbitos ecológico, social y económico (Cantú-Martínez, 2015). Estas ideas, que dan cuenta del camino hacia una dirección integradora, que ha sido además coincidente y donde se ha permitido un diálogo que aborda los distintos conocimientos disciplinares con la finalidad de orientar un nuevo tipo de desarrollo socioeconómico y ecológico, que hoy conocemos como desarrollo sustentable.

El propósito del presente trabajo es contribuir al esclarecimiento de los desafíos de la educación para impulsar la sustentabilidad. Desde un contexto pormenorizado, donde se acoge documentación esencial con la finalidad de establecer la relación entre estas dos variables, y posicionarlas para construir valores y actitudes sociales, que promuevan estas conductas en las personas y que les permitan alcanzar un estilo de vida de orden sustentable.

Para esto, se transitará por una revisión de la información relativa a la sustentabilidad y su relación con la educación. Por otra parte, se discurrirá sobre la contribución de la educación para el logro de la sustentabilidad y el papel de las instituciones educativas para alcanzar esto. Posteriormente, se observarán los retos que enfrenta la educación para la sustentabilidad y, por último, realizar unas consideraciones al respecto.

\section{Marco conceptual}

Cantú-Martínez (2018) comenta que en el presente subsistimos

en un período en el que se suscitan cambios a nuestro alrededor de manera vertiginosa. 
Particularmente en las tres últimas décadas, producto del progreso en la ciencia y tecnología, sobre todo en las áreas de robótica, informática, comunicaciones $\mathrm{y}$, esencialmente, en el ámbito de la biomedicina. Estos avances nos han permitido explorar el espacio exterior y examinar dimensiones menores a las del átomo. Sin embargo, este supuesto avance ha creado serias dificultades (p. 42).

Estas eventualidades, se han evidenciado mediante el cambio climático, incendios forestales, desertificación, vertimiento de residuos al suelo, aire y agua. Contextos que nos permiten explicar el deterioro de su calidad. Además de la disminución de los recursos naturales renovables y no renovables, de los cuales disponemos para nuestra existencia, se han presentado altos costos ecológicos como socioeconómicos, tanto localmente como de manera global (Cantú-Martínez, 2014).

Por ello, la educación se constituye en el artificio ideal para reorientar y revertir las deplorables crisis de índole social como ecológica que subsiste en el seno de nuestra sociedad y en el entorno que nos circunda. En este sentido, se yergue el pronunciamiento que Orr (2004) hace al mencionar que toda dificultad actual tiene su origen en las precepciones e ideas erróneas que subsisten en los seres humanos, por lo cual no se puede atribuir el momento actual como un acontecimiento generado por la carencia de educación, pero si es un aspecto que la educación debe atender.

Concebido lo anterior, comenta Delgado (2002), que

si consideramos el problema ambiental como un problema de la relación humana con su entorno natural es necesario encontrar fundamentos universales que nos permitan formar un tipo de ser humano distinto, capaz de cambiarse a sí mismo y plantearse una nueva relación con su entorno [...] producir el cambio de conciencia debería conducir por sí mismo al cambio de actitud social, espiritual y material para con el entorno (p. 7).

Estos preceptos, anteriormente mencionados, fueron suscritos en la Declaración de Estocolmo en 1972, particularmente en el Principio 19 que detalla entre sus líneas lo siguiente:

Es indispensable una labor de educación en cuestiones ambientales, dirigida tanto a las generaciones jóvenes como a los adultos y que preste la debida atención al sector de población menos privilegiado, para ensanchar las bases de una opinión pública bien informada, y de una conducta de los individuos, de las empresas y de las colectividades inspirada en el sentido de su responsabilidad en cuanto a la protección y mejoramiento del medio ambiente en toda su dimensión humana (Naciones Unidas, 1972, Principio 19).

Esta declaración ocasionó que en lo subsecuente se llevaran a cabo una serie de reuniones y declaraciones, en derredor de la educación (ver Tabla 1), con la finalidad de ir edificando un nuevo marco social que hoy en día tiene como intención dar cumplimiento a la Agenda 2030 (Cantú-Martínez, 2016; Cantú-Martínez, 2018).

En la tabla 1 se aprecian los encuentros y declaraciones que la colectividad académica ha realizado en el mundo para hacer tangible la sustentabilidad mediante la educación.

En relación con la Agenda 2030, que fue aceptada universalmente en septiembre del 2015, se dieron a conocer los 17 Objetivos del Desarrollo Sustentable (ODS) que se encuentran vinculados a este documento, y que orienta las acciones y demarca las metas a conseguir en el marco del desarrollo sustentable, como lo consigna la Comisión Económica para América Latina y el Caribe (CEPAL, 2016). Todas estas acciones están encaminadas a revertir “la visión dicotómica y opuesta -entre sí- del mundo natural y el social, lo que condicionó la producción de un medio ambiente en permanente destrucción en tiempo histórico" (Bayón, 2016, p. 54). Cabe señalar también, según Maldonado (2018), que se busca contrarrestar los efectos de las siguientes visiones:

1. Antropocéntrica: heredada de la visión judeocristiana, en la que el ser humano es considerado como ajeno y superior a la naturaleza.

2. Razón y dominio: el ser humano renuncia 
incluso a sí mismo por la presión ejercida por la sociedad de consumo.

3. Reduccionista: simplifica de forma excesiva el mundo y la vida, lo que impide analizar las relaciones sistémicas y complejas entre la sociedad y la naturaleza.

4. Irresponsable: la supuesta neutralidad de nuestros actos y la falta de conciencia sobre sus consecuencias.

5. Utilitarista: considera la naturaleza como un bien inagotable y pondera las necesida- des por encima de los recursos.

6. Economicista: el dinero es el único criterio de valor (p. 15).

Por lo tanto, la Agenda 2030 deja entrever que el ser humano es un ser biológico, de constitución social y finalmente con un marco cultural en el que se han conjugado esencialmente estos aspectos en el discurso de la sustentabilidad para emprender una nueva afinidad social, un renovado contexto de convivencialidad, como también de subsidiaridad (Santos, 2011).

Tabla 1

Reuniones y declaraciones para contribuir a la educación sustentable.

\begin{tabular}{|c|c|c|}
\hline Lugar y fecha & $\begin{array}{l}\text { Reunión/ } \\
\text { Documento }\end{array}$ & Contribución \\
\hline Belgrado/octubre 1975 & Carta de Belgrado & $\begin{array}{l}\text { Promulgó el establecimiento de una nueva } \\
\text { relación con el ambiente y entre las personas. } \\
\text { Se pronunciaron favor de la educación } \\
\text { ambiental para lograr esta aspiración. }\end{array}$ \\
\hline Tbilisi/octubre 1977 & Declaración de Tbilisi & $\begin{array}{l}\text { - Se pronuncia a favor de la educación para } \\
\text { resolver las problemáticas ambientales, desde } \\
\text { una perspectiva regional e internacional. }\end{array}$ \\
\hline Moscú/agosto 1987 & Encuentro de Moscú & $\begin{array}{l}\text { Acuerdan llevar a cabo una estrategia } \\
\text { internacional de educación ambiental, y } \\
\text { establecerla en la década de los noventas. }\end{array}$ \\
\hline Jomtien/marzo 1990 & $\begin{array}{l}\text { Declaración Mundial Sobre } \\
\text { Educación Para Todos }\end{array}$ & $\begin{array}{l}\text { Se establecieron lineamientos para orientar y } \\
\text { mejorar los servicios de educación básica. }\end{array}$ \\
\hline Talloires/octubre 1990 & Declaración de Talloires & $\begin{array}{l}\text { - Se esclareció la contribución que deberían } \\
\text { llevar a cabo las instituciones de educación } \\
\text { superior con la finalidad de promover la } \\
\text { sustentabilidad. }\end{array}$ \\
\hline Río de Janeiro/junio 1992 & Cumbre de La Tierra & $\begin{array}{l}\text { - Se reconoce universalmente a la educación } \\
\text { como un soporte relevante para fomentar el } \\
\text { desarrollo sustentable. }\end{array}$ \\
\hline Salónica/diciembre 1997 & Declaración de Salónica & $\begin{array}{l}\text { - En esta proclama se hace mención que para } \\
\text { lograr la sustentabilidad la educación se } \\
\text { constituye en el artificio para alcanzar esta }\end{array}$ \\
\hline Dakar/abril 2000 & Marco De Acción de Dakar & $\begin{array}{l}\text { - Establece que la educación es un derecho } \\
\text { humano fundamental y es clave para el } \\
\text { desarrollo sustentable }\end{array}$ \\
\hline $\begin{array}{l}\text { Johannesburgo/ } \\
\text { septiembre } 2002\end{array}$ & $\begin{array}{l}\text { Cumbre Mundial sobre } \\
\text { Desarrollo Sostenible }\end{array}$ & $\begin{array}{l}\text { - Se establece Decenio de las Naciones Unidas } \\
\text { de la Educación para el Desarrollo Sostenible } \\
(2005-2014)\end{array}$ \\
\hline Mascate/mayo 2014 & Acuerdo de Mascate & $\begin{array}{l}\text { - Se comprometen que todos los estudiantes } \\
\text { llegarán al } 2030 \text { con las competencias y } \\
\text { actitudes sobre desarrollo sustentable }\end{array}$ \\
\hline Incheon/mayo 2015 & Declaración de Incheon & $\begin{array}{l}\text { - Se reconoce a la educación para el logro de los } \\
\text { objetivos de desarrollo sustentable }\end{array}$ \\
\hline
\end{tabular}

Nota: Elaborado a partir de Cantú-Martínez (2018). 


\section{Nuevo contrato social: sustentabilidad y educación}

Se reconoce en la educación el instrumento que permite a nuestra sociedad desarrollar tanto la capacidad intelectual, como también el segmento moral y de carácter emotivo en relación con los patrones y marcos de referencia cultural que se posean. En otras palabras, se transforma a la persona mediante la transmisión de conocimientos, ya que todo ser humano está marcado por los rasgos culturales que trae consigo (Molano, 2012).

Esto posiciona a la educación en un proceso humanizado y bastante complejo culturalmente. Pero detrás de este proceso subsiste un legítimo propósito que es examinar y perpetuar aquellas condiciones que favorezcan y merezcan ser trasmitidas para la formación y seguridad de todo ser humano, y así hacerlo libre de forma intelectual.

Recordemos que el ser humano es producto del mismo ser humano, es decir, como indica León (2007) a la vez que se concibe de manera individual también lo hace de manera colectiva, construye diferentes concepciones de lo que le rodea, así como los estilos de vida que puede ostentar y con ello, compararse con la otredad. Evento que a su vez da origen a su propia autodeterminación.

Anotaremos que de acuerdo con Cantú-Martínez (2008) esto se consigue flagrantemente mediante la educación al

(1) liberar y dignificar a las personas, al edificar valores más profundos que se vinculen con la conciencia; (2) impulsar cambios en la sociedad para que esta se constituya más íntegra y justa; (3) perfeccionar las potencialidades de todas las personas; (4) aleccionar a los individuos para el trabajo y (5) desarrollar entre las colectividades humanas la sociabilidad y el respeto por la diversidad de culturas (p. 42).

Por lo tanto, la educación ocupa un lugar preponderante en la sociedad, ya que mediante esta, toda colectividad social reconoce una panorámica de su entorno como también de la vida -incluso de su propia vida- que denota una manera de pensar y así resolver las eventualidades y sus necesidades, como lo asevera León (2007).

Consecuentemente, la educación se yergue como la búsqueda del bien y de lo que es adecuado para el bienestar de las personas, pero sobre todo es un intento humano de carácter racional que pretende perfeccionar a toda sociedad y persona. De esta manera Cantú-Martínez (2008) hace referencia que la educación ha sido el instrumento social mediante el cual toda sociedad permuta sus esquemas y enfoca sus esfuerzos para allegarse una vida mejor.

En esta línea discursiva, señala Guzmán (2011), que una "educación impartida en un marco que preste atención educativa a los problemas humanos y sociales, permite la transformación de sus miembros y les otorga las herramientas para que potencien su desarrollo cultural y, en consecuencia, se produzca su integración social" (p. 112).

La educación, como se ha podido observar, es un instrumento verdaderamente virtuoso para la sociedad humana. En distintos momentos de orden histórico, la educación se ha erguido como el dispositivo capaz de permitir la integración de una nueva sociedad o bien ha reforzado los ámbitos de una identidad entre las personas. Con esto en mente, evocamos lo mencionado por Barrios (2008), que comenta que la educación es, en primera instancia, una actividad inseparable del progreso y desarrollo de ser humano y ,en segunda instancia, que a través de ella le permite desenvolver sus capacidades, trazar sus proyectos de vida y civilizarse.

Con lo cual, se pretende reorientar y reconfigurar la postura de la conducta humana, considerando que la práctica del ejercicio cotidiano de un ser humano se encuentra fuertemente influida por su postura interior, y que de acuerdo con Martí (2005) obedece a

a. La intencionalidad (los pensamientos son creencias).

b. La conciencia (la experiencia implica una subjetividad).

c. La intimidad (los estados mentales son personales o únicos)

d. La continuidad (la subjetividad fluye en un yo unificado).

e. La selectividad (la mente sólo atiende a determinados rasgos del mundo).

Como se observa, la educación se erige como un bien social que, de manera muy influyente, contribuye al progreso de las sociedades y de todo ser humano. Esencialmente al proporcionarle co- 
nocimientos y todos aquellos elementos que nos determinan, describen e identifican como seres humanos. Por lo tanto, se puede indicar que el cometido de la educación es impulsar el desarrollo integral y ordenado de toda persona.

\section{Las instituciones de educación en la sustentabilidad}

El rol que las instituciones de educación tienen en el marco del desarrollo sustentable, es sumamente relevante. Esto surge, cuando desde una perspectiva de carácter pedagógico, en estos recintos escolares confluyen tanto intereses particulares que emergen legítimamente de las personas que se educan, como también los de orden público y social que se transcriben en los educandos, mediante los procesos de enseñanza aprendizaje por parte de las instituciones receptoras (Retamoso, 2007).

Es así, que en este escenario de interacción se vislumbra lo que Díaz (como se citó en Retamoso, 2007) denomina como la pedagogización de la vida cotidiana, en la que se relacionan y unifican los pareceres personales con las prácticas institucionales y las normas de orden social. Es en esta vinculación que se da en el espacio educativo, donde se observa el proceso de desarrollo de los ciudadanos, en el cual convergen inclinaciones personales como públicas

De aquí también se desprenden las estructuras de la sociedad y la formación ciudadana, que se da en una dialéctica educativa, donde interaccionan los educandos con el contexto de la realidad en la que se "analiza, comprende, interpreta y trasforma los problemas reales que afectan a una comunidad en particular [...] [donde la] educación [se instaura] como posibilidad para la identificación de problemas y para la búsqueda de alternativas de solución" (Ramírez, 2008, p. 109).

Por lo anteriormente mencionado, Retamoso (2007), alude que la pedagogización de la vida cotidiana, es la postura ideológica que se exhibe frente al mundo y determina la manera de valorarlo como de comprenderlo. Para Pierre Astolfi (como se citó en Mella, 2003) una educación que lleve a los educandos a instruirse y educarse cabalmente vislumbra tres momentos para alcanzar un aprendizaje agudo y consciente. El primer momento, consta de recibir información, el segundo momento, que esta información genere conocimiento y finalmente como tercer momento, que este conocimiento produzca saber en el educando, para permitirle organizar las experiencias que se suscitan tanto dentro del aula como fuera de ella.

Es así que las instituciones educativas requieren formar una persona que pueda evaluar los ajustes pertinentes que requiere hacer en su vida, como también reflexionar en las habilidades que debe fortalecer y desarrollar, así como discernir los conocimientos que debe tener para emprender nuevas tareas (Ambrosy, 2015); por este motivo, estas instancias institucionales deben velar por la formación de un ser humano con un criterio histórico y examinador, que le permita desentrañar de manera retrospectiva todo lo acontecido en el pasado, como también le conceda examinar el presente a la luz del conocimiento actual y le dé argumentos para prever el futuro mediante el discernimiento de las problemáticas que enfrenta, tanto colectivamente como individualmente.

En este proceso se puede identificar claramente el marco de la sustentabilidad al cual se aspira, que es formar seres humanos conscientes y a quienes además se le ceda genuinamente el saber, con el propósito de resignificar los imaginarios simbólicos y le permita enlazar el contexto histórico, el sociocultural y el de convivencia colectiva. Todo esto, debe darse en un continuo educativo, desde los niveles más básicos hasta el superior, por el cual transite todo ser humano de manera formal. Además, este recorrido educativo debe ofrecerles a las personas una mirada global de la situación en que se subsiste, con la finalidad de reorientar su pensar y actuar hacia la sustentabilidad. En ese sentido, para ampliar los espacios educativos a lo largo de la vida de una persona, debe existir una armonización entre la educación formal con la no formal.

Agregando en esta misma línea discursiva, la secuencia de la formación educativa de un ser humano bajo el marco de la sustentabilidad, requiere que en ello subsistan saberes distintos que son necesarios -como los de ahora-, pero estos deben estar aunados a la historia y conocimientos anteriores que se les ha impartido, con la finalidad de que al conjugarse los habiliten para pensar y racionalizar su realidad de manera distinta (Southwell, 2013). 
Por esta razón se alude que, en este nuevo escenario las Instituciones de Educación Superior (IES) particularmente, deben incidir a un nivel de comunidad, teniendo como primer contacto a los educandos. A ellos se les formará con recursos humanos competentes y deberán estar preparados para resolver problemáticas sociales; como segundo término cuentan con el escenario del servicio social, donde sus estudiantes llevan a cabo prácticas profesionales que los acercan y sensibilizan de las necesidades comunitarias y ambientales. Y finalmente, cuentan con el capital intelectual para la realización de proyectos de investigación e intervención que pueden incidir en la resolución de las eventualidades socioambientales y que coexisten en el entorno donde estas inciden.

En lo anterior se ponen de manifiesto las competencias específicas en los educandos, las cuales se clasifican en tres vertientes: las académicas, las disciplinares y las profesionales. Pérez, Julián y López (2009) señalan que estas confieren a los estudiantes lo siguiente:

Competencias académicas: son las que se relacionan directamente con los conocimientos teóricos que tradicionalmente se han evaluado y calificado (saber).

Competencias disciplinares: son todos aquellos conocimientos prácticos que relacionan los teóricos, tan necesarios para el desarrollo profesional, y que son muy valorados en el mercado laboral (hacer).

Competencias profesionales: son aquellas que incluyen tanto habilidades de comunicación como las de indagación; pero sobre todo las de saber realizar en la puesta en práctica profesional (saber hacer) (p. 24).

Al mismo tiempo las IES precisan hacer ajustes de sus estructuras organizacionales, así como renovar las relaciones con la sociedad, concretamente en el ámbito de la gestión e intervención social (Montaño \& Solís, 2001). Como consecuencia las IES se deben constituir en medios que permitan aglutinar el campo del saber y la práctica, donde según Kant (como se citó en Villavicencio,2008), comenta que los centros educativos de estudios superiores son los recintos donde se cristalizan las ideas, se conciben los pensamientos y se organiza el conocimiento de la sociedad, y además es don- de se asigna para cada disciplina un profesor que constituiría y edificaría una entidad científica.

Con ello se llega a la pregunta ¿cuál debería ser la tarea de las IES para instituir y circunscribir el conocimiento de la sustentabilidad en la sociedad? Sin lugar a dudas, en primera instancia sería la búsqueda de la verdad, y en segundo término de acuerdo con Nieto y Medellín (2007) intervenir en

a. Debates teóricos y conceptuales sobre medio ambiente y sustentabilidad.

b. Estudios de evaluación, diagnóstico, prevención, control y restauración.

c. Procesos de consulta pública, participación social, resolución de conflictos y generación de consensos.

d. Procesos de información y capacitación.

e. Nuevos medios de comunicación electrónica (publicaciones, grupos virtuales, etcétera).

f. Desarrollo de métodos y tecnologías.

g. Formulación de criterios y propuestas en las políticas públicas y en la gestión ambiental (p. 38).

Por este motivo, las IES en este contexto de la sustentabilidad se han erguido como instancias, que se personifican ante la sociedad humana, de actualidad e innovación del conocimiento, donde se articula además la modernidad con la razón y lo socialmente colectivo. Bajo esta misma perspectiva Torres (2012) también señala que las IES deben

1) Socializar la enseñanza y colaborar en el diseño de políticas que multipliquen las oportunidades de aplicación del conocimiento.

2) Definir agendas de investigación que atiendan necesidades sociales.

3) Promover la aplicación de los resultados.

4) Atender desde los criterios de evaluación académica los problemas del subdesarrollo.

5) Mantener la excelencia de los resultados de investigación (p. 69).

Dentro de este contexto, Malinowski (como se citó en Iglesias, 2007) comenta que para

preservar y propiciar la evolución de la cultura se requiere de la educación, y para lograr los fines se crean las instituciones como unidades de organización. Las instituciones tienen como fundamento series de valores alrededor de los cuales los seres humanos se congregan (p. 48). 
Por ello, Torres (2012) alude que las IES enfocadas en la sustentabilidad también deben ocuparse de renovar los ambientes de la enseñanza superior, adaptar los currículos académicos a las necesidades sociales, ofertar espacios de formación profesional y continua para individuos de distintos grupos etarios, aumentar la oferta educativa, permitir que los académicos e investigadores de distintas especialidades se agrupen para trabajar vinculadamente para la discernimiento y resolución de las eventualidades sociales como ambientales, entre otros aspectos; y así cumplan las IES su papel social y ambiental de manera responsable.

\section{Desafíos en la educación para la sustentabilidad}

La educación siempre ha estado presente en la trayectoria de vida del ser humano, tan solo basta citar que en las comunidades primitivas que nos antecedieron las "relaciones directas entre los miembros del clan y de las pequeñas comunidades se [reconoce que se] produce la transmisión natural de prácticas de conducta de padres a hijos, entre diferentes generaciones, relativas al nexo [del ser humano con su entorno natural]" (Hernández, 2014 , p. 10). Así es como comienza el respeto y el reconocimiento del valor de la naturaleza. Sin embargo, este conocimiento y actitud se fue extraviando en la medida que se encontró en el medio ambiente un contexto para comercializar sus elementos y buscar acceder a otros beneficios materiales.

Hoy en día la educación debe permitir el acceso de cada ser humano a las competencias para la sustentabilidad, que Aznar, Ull, Martínez y Piñero (2014), precisan como la conglomeración constituida de saberes, prácticas, posturas y significados que las personas poseen y las aplican en distintos ámbitos sociales, laborales, educativos y familiares, para solucionar los problemas socioambientales y así transfigurar la realidad con razonamientos que induzcan la sustentabilidad.

Esta educación, conforme a Aznar y Ull (2009) debe estar acompañada de diferentes tipos de racionalidad que son: "la racionalidad teórica, la racionalidad práctica y la racionalidad ética" (p. 221). Donde la racionalidad teórica nos da acceso a la aplicación de todo saber científico para mejorar el ejercicio y acto de la educación. Mientras la racionalidad práctica, nos permitiría ocuparnos en la construcción de nuevo conocimiento a partir de la observación, experiencia y retroalimentación, y así permitir orientar nuestras posturas como sociedad. Finalmente, la racionalidad ética, nos permitirá recrear nuevas configuraciones desde la integración del conocimiento teórico y práctico, mediante una actitud reflexiva.

En este sentido Novo (2009), menciona que en la vida comunitaria es fundamental que la educación provea las respuestas lícitas ante los desafíos que se le plantean a la sociedad humana. Por lo tanto, este tipo de educación procura la edificación de una ciudadanía comprometida a la cual suministra instrumentos para resolver las eventualidades, impulsando así actuaciones responsables, tanto de manera colectiva como individual (Leal Filho, 2009).

Esta educación debe combatir, en el marco de las ideas, las configuraciones del imaginario que han creado los tutores del crecimiento económico. El cual se caracteriza por ser resueltamente agresivo y que se ha mantenido a costa de la inmolación del entorno natural, donde además solo ellos se adjudican una condición de racionalidad, y de la cual privan a todos sectores sociales ajenos a ellos. De esta manera arbitraria, sobreponen sus planteamientos, y como hemos podido advertir, ha robustecido las asimetrías sociales entre los seres humanos (Elizalde, 2009).

Este modelo de educación también debe estar asentado en el paradigma socio-crítico, que esboza una racionalidad que amalgama juicios, valores e intereses de carácter social, como también de un compromiso para la transformación desde el seno de la misma sociedad (Alvarado \& García, 2008); por lo cual, esta ostenta una palpable representación autoreflexiva. Cuando nos referimos a este modelo socio-crítico, es necesario hacer mención de Popkewitz, que en 1988 (como se citó en Alvarado \& García, 2008) comenta que este modelo debe contar con los siguientes principios: “(a) conocer y comprender la realidad como praxis; (b) unir teoría y práctica, integrando conocimiento, acción y valores; (c) orientar el conocimiento hacia la emancipación y liberación del ser humano; y (d) proponer la integración de todos los participantes" (p. 190).

Dentro de este contexto, Alvarado y García (2008) plantean que para Habermas, el conoci- 
miento es emanado de las preocupaciones que día a día emergen de forma personal o bien colectivamente, y siempre está asociado a las necesidades de la especie humana, donde este está vinculado permanentemente a las condiciones históricas y sociales del ser humano.

De ello resulta, que la presencia de la reflexión, del cuestionamiento y la toma de acuerdos se constituyan para todo ser humano o colectivo social en el fundamento que les otorga un conjunto de ideas, percepciones y valores. Con ello apreciarán y estimarán el entorno social al cual están adheridos y en el que intervendrán a través de sus opiniones y acciones justificadas. Por este motivo, la educación retoma suma importancia para la sustentabilidad, pues orienta estas voces y actividades para el mejoramiento de la calidad de vida de todo ser humano (De Vincezi \& Tudesco, 2009).

Es así, que en el marco de los ODS y sus metas que constituyen 169 , se fincan los retos y logros que se esperan para el período del 2016 al 2030. Estos retos, ansían contrarrestar la miseria, el hambre, la erradicación de padecimientos considerados como curables, proporcionar agua limpia para todos y una educación para todo ser humano, entre otros aspectos. De manera particular, el Objetivo 4, de los ODS (CEPAL, 2016), refiere a garantizar una educación inclusiva, equitativa y de calidad, como también el promover oportunidades de aprendizaje durante toda la vida para todos. De tal manera, se contempla a la educación como el pilar para mejorar la vida de todo ser humano y le permita contar con conocimientos pertinentes para el desarrollo de soluciones a las eventualidades -sociales y ambientales- más apremiantes, y por lo tanto contribuir al desarrollo sustentable, lo cual se traduce en muchos desafíos.

Sin lugar a dudas, estos retos que se han planteado nos redirigen como sociedad, a mejorar las pautas y trayectorias anteriores, con la finalidad de dar génesis en el marco de la sustentabilidad, a un espacio educativo renovado que tiene como puntos medulares los valores, la ética y el humanismo. Y como señala Retamoso (2007), es mediante la educación la forma en que todo colectivo social conjuntamente con su cultura va logrando superar los momentos más aciagos, para edificar un futuro promisorio para las nuevas generacio- nes. Es así que mediante la educación y las metas del Objetivo 4, se pretende configurar un nuevo ser humano en el marco de la sustentabilidad, de tal manera que permita con esto construir, deconstruir y reconstruir el tejido social. Especialmente a la luz del examen crítico, del uso de razonamientos integrales, como de deliberaciones colaborativas moderadas por un sentido de compromiso tanto hacia las generaciones actuales como a las próximas por venir (Caride, 2017).

Esto es de suma relevancia, ya que se acepta mundialmente que una de las características que distinguen a los países en vías de desarrollo, subdesarrollados e infradesarrollados, con respecto a los desarrollados, es la falta de una educación aceptable o bien, la trayectoria educativa inconclusa con la que cuentan muchas personas. Esto, sin dejar de lado a los paises en los que persiste aún una actividad económica de tipo colonialista y dependiente substancialmente de la venta y exportación exclusivamente de materias primas, que conlleva consigo un extractivismo exacerbado llevado a cabo por las naciones desarrolladas, y que no reditúa ningún beneficio para las naciones proveedoras de estas materias primas (Torres, 2012).

\section{Conclusiones}

La educación que se compromete con la sustentabilidad, sin lugar a dudas conlleva implícitamente la búsqueda de empoderar y mejorar las condiciones de vida de toda persona. Particularmente porque contribuye a que cada individuo obtenga los conocimientos, los valores y las destrezas prácticas para actuar de manera responsable, con la convicción de generar en la colectividad social un estilo de vida reorientado hacia la equidad y justicia social, preceptos que subyacen al desarrollo sustentable.

Se recuerda además, de acuerdo con Southwell (2013), que dentro de las instituciones educativas es donde se vertebra tanto las creencias y acciones de todos los enclaves generacionales, etnográficos, culturales y religiosos, pero donde además se puede insertar los fundamentos de la sustentabilidad.

Por otra parte, esta novel educación encaminada a la sustentabilidad se instrumenta y fun- 
damenta en el razonamiento. De esa manera se llevan a cabo acciones concretas de consumo responsable y de contribuir en las políticas sociales mediante una participación ciudadana activa, donde se muestra lo importante que es la incorporación de la dimensión ética. Enlazando así las diferentes formas de conocimiento, y que tiene especialmente en las IES, el envión para impulsar el conocimiento de la sustentabilidad y el mejoramiento de las condiciones de vida.

Con ello, se otorga un punto de vista de orden interdisciplinar, produciendo así en el educando un pensamiento que lo guíe a una reflexión crítica. De acuerdo con esta aseveración, permite encontrar la congruencia que debe erguirse entre la realidad social y el conocimiento que media en los recintos educativos donde son edificadas las personas en ciudadanos. Redondeando esta noción, se alude también, que es en estos espacios educativos, especialmente los universitarios, en donde todo ser humano experimenta y examina numerosos valores, que en el contexto de la educación le permiten discernir y jerarquizarlos. Esto les permitirá a las personas la posibilidad de dirimir los distintos conflictos que pueden surgir de una decisión, y que proceden de todo acto de valoración, en el que además los preceptos de la sustentabilidad sean internalizados en el individuo, sin la presencia de alguien o algo que se lo indique (Iglesias, 2007).

En apego a lo anterior, se podría hablar así de una ciudadanía alfabetizada no solo en el ámbito social y económico, sino orientada también a la resolución de las eventualidades ambientales. Caracterizada por opinar y colaborar en tópicos sociales que atañen aspectos como el revertir la pobreza, promover la paz social, respetar los derechos humanos y particularmente conducirse de acuerdo a las exigencias necesarias para procurar y conservar el entorno natural, otorgándole una valoración justa a la naturaleza. De tal manera, que este es el extraordinario espacio de la educación para la sustentabilidad, donde se manifiestan todas las facultades virtuosas y carencias de la naturaleza humana, pero invariablemente bajo la reflexión del aprendizaje (Lamata, 2003).

Aprendizaje que requiere de la expresión ver- bal y de un entendimiento interactivo para percibir aquellos sucesos que sobrepasan la propia experiencia de los posibles interlocutores, donde se privilegie el participar las experiencias y las interpretaciones resultantes de lo que designamos como sustentabilidad, y acercarnos mediante acuerdos, al pretendido conocimiento de lo que es el desarrollo sustentable.

Basándose en lo anterior, toma mucha relevancia en este momento lo que Castells (2005) comentaba al indicar que la sociedad de hoy en día se sustenta en el accionar del conocimiento, el cual se aplica en ella misma y por lo cual se constituye así en la medular fuente de productividad y trasformación del panorama socioambiental. Con esto evoca, que tras todo conocimiento subsiste un proceso de educación de las personas y la incursión del conocimiento de la sustentabilidad en la sociedad no es ajeno a ello. Recordemos que la educación es la restructuración de la actitud de toda persona, la cual cuenta con la capacidad de transformarse y cambiar.

Por último, considerando la anterior premisa, esperamos que esto nos conduzca a un nuevo proyecto de humanismo donde toda aprehensión de la realidad sea integradora y totalizadora, cómo lo requiere el concepto de sustentabilidad en esta época, y dignifique así la vida de todo ser humano y todo aquello que le envuelve. Reconozcamos, que toda sociedad que nos antecedió tuvo una oportunidad histórica en un período de tiempo determinado, en el que se apeló a la priorización de ciertos valores y habilidades para su continuidad.

A esto Foucault (como se citó en Márquez, 2014), le denominó a priori histórico, que entraña un trasfondo de orden intelectual que estipula tanto el pensamiento y las actividades del ser humano, y que se sustenta en las experiencias anteriores. Ahora bien, pareciera que por lo antes mencionado, que quizás nuestro tiempo como sociedad posmoderna ha llegado y para rectificar nuestro camino, como también garantizar la continuidad de la sociedad humana, esta priorización se llama hoy en día desarrollo sustentable. El cual deberá traducirse en modos de vida que expresen la sustentabilidad y para ello es imprescindible la alteridad en la educación. 


\section{Referencias}

Alvarado, L. \& García, M. (2008). Características más relevantes del paradigma socio-crítico: su aplicación en investigaciones de educación ambiental y de enseñanza de las ciencias realizadas en el Doctorado de Educación del Instituto Pedagógico de Caracas. Sapiens Revista Universitaria de Investigación, 9(2), 187-202. Recuperado de https://tinyurl.com/yxtbnllg

Ambrosy, I.L. (2015). La educación en el contexto de la sociedad actual. Revista Calidad en la Educación Superior, 6(2), 200-213. Recuperado de https://tinyurl. $\mathrm{com} / \mathrm{y} 3 \mathrm{~d} 85 \mathrm{kog}$

Aznar, P. \& Ull, M.A. (2009). La formación de competencias básicas para el desarrollo sostenible: el papel de la Universidad. Revista de Educación, (No. Extraordinario), 219-237. Recuperado de https://tinyurl.com/ y53yyj9j

Aznar, P., Ull, M.A., Martínez, M. del P. \& Piñero, A. (2014). Competencias básicas para la sostenibilidad: un análisis desde el diálogo disciplinar. Bordón, 66(2), 2014, 13-27. https://doi.org/10.13042/Bordon.2014.66201

Barrios, M. (2008). Por un continuo educativo en clave de desarrollo humano. Una visión desde Fe y Alegría. En: J.F. Bruni Celli, N. Aguirre Ledezma, F.J .Murillo Torrecilla, H. Díaz Díaz, A. Fernández Ludeña \& M. Barrios Yaselli (Autores). Una mejor educación para una mejor sociedad. Propuestas para el diálogo y la transformación educativa en América Latina y el Caribe. (pp. 7-34). Madrid: Federación Internacional de Fe y Alegría. Recuperado de https://tinyurl.com/y3eqxcao

Bayón, P. (2016). La percepción de riesgo por peligros hidrometeorológicos extremos en Cuba: mirada desde el entorno geográfico. Trayectorias, 18(43), 53-72. Recuperado de https://tinyurl.com/y5yw4yht

Beck, U. (1998). La sociedad del riesgo, hacía una nueva modernidad. Barcelona: Paidós. Recuperado de https://tinyurl.com/yyq57qss

Cantú-Martínez, P.C. (2008). Desarrollo Sustentable: Conceptos y Reflexiones. México: Ed. Universidad Autónoma de Nuevo León. Recuperado de https://tinyurl.com/yyb5w73u

Cantú-Martínez, P.C. (2014). Educación ambiental y la escuela como espacio educativo para la promoción de la sustentabilidad. Revista Electrónica Educare, 18 (3), 39-52. https://doi.org/10.15359/ree.18-3.3

Cantú-Martínez, P.C. (2015). Desarrollo Sustentable. Antes y Después de Río +20. México: Universidad Autónoma de Nuevo León y Organización Panamericana de la Salud. Recuperado de https://tinyurl.com/y2tpfpwp Cantú-Martínez, P.C. (2016). Los nuevos desafíos del desarrollo sustentable hacia 2030. Ciencia UANL, 19(78), 27-32. Recuperado de https://tinyurl.com/y44fze8q Cantú-Martínez, P.C. (2018). El movimiento educativo y su contribución a la sustentabilidad. Ciencia UANL, 21(91), 42-50. Recuperado de https://tinyurl.com/yy36undd

Caride, J.A. (2017). Educación social, derechos humanos y sostenibilidad en el desarrollo comunitario. Teoría de la Educación, 29(1), 245-272. https://doi.org/10.14201/ teoredu291245272

Castells, M. (2005). La era de la información. Economía, sociedad y cultura. La sociedad red Vol. 1. Buenos Aires: Siglo XXI Editores. Recuperado de https://tinyurl.com/y6ym4eph

Comisión Económica para América Latina y el Caribe (CEPAL) (2016). Agenda 2030 y los Objetivos de Desarrollo Sostenible. Una oportunidad para América Latina y el Caribe. Santiago: Naciones Unidas/ CEPAL. Recuperado de https://tinyurl.com/y3ch58hr

De Vincezi, A. \& Tudesco, F. (2009). La educación como proceso de mejoramiento de la calidad de vida de los individuos y de la comunidad. Revista Iberoamericana de Educación, 49(7), 1-12. Recuperado de https://tinyurl.com/y5bhl34s

Delgado, C.J. (2002). Los límites socioculturales de la educación ambiental. México: Siglo XXI. Recuperado de https://tinyurl.com/y6g2uvdn

Elizalde, A. (2009). ¿Qué desarrollo puede llamarse sostenible en el siglo XXI? La cuestión de los límites y las necesidades humanas. Revista de Educación, (No. Extraordinario), 53-75. Recuperado de https://tinyurl.com/y2gt2ega

Guzmán, M. (2011). Sociedad y educación: la educación como fenómeno social. Foro Educacional, 19, 109. 120. Recuperado de https://tinyurl.com/y5e9cgmb

Hernández, J. M. (2014). Educación Ambiental y vida sostenible en la Historia. En: J. M. Hernández Díaz (Coord.). Historia y Presente de la Educación Ambiental. Ensayos con perfil iberoamericano. (pp. 9-32). Salamanca: FahrenHouse. Recuperado de https://tinyurl.com/yytk64wt

Iglesias, L. (2007). La cultura contemporánea y sus valores. España: Anthropos. Recuperado de https://tinyurl. com/y3zhpddx

Lamata, (2003). Fuentes educativas. En: R. Domínguez \& R. Lamata (Coords.). La construcción de procesos for- 
mativos en educación no formal. (pp. 23-56). Madrid: Narcea. Recuperado de https://tinyurl.com/y22147un

Leal Filho, W. (2009). La educación para la sostenibilidad: iniciativas internacionales. Revista de Educación, (No. Extraordinario), 263-277. Recuperado de https://tinyurl.com/y53yyj9j

León, A. (2007). Qué es la educación. Educere, 11(39), 595 604. Recuperado de https://tinyurl.com/y54kbmha

Maldonado, T.N.J. (2018). Educación ambiental para la sustentabilidad. Didac, 71, 13-20. Recuperado de https://tinyurl.com/y6lthgdj

Márquez, J.W. (2014). Michael Foucault y la contra-historia. Historia y Memoria, 8, 211-243. https://doi. org/10.19053/20275137.4445

Martí, E. (2005). Desarrollo, cultura y educación. Buenos Aires: Amorrortu. Recuperado de https://tinyurl.com/ yxqqkwqn

Mella, E. (2003). La educación en la sociedad del conocimiento y del riesgo. Revista Enfoques Educacionales, 5(1), 107-114. Recuperado de https://tinyurl.com/ y3uahmzl

Molano, A. B. (2012). La complejidad de la educación ambiental: una mirada desde los siete saberes necesarios para la educación del futuro de Morin. Revista de Didáctica Ambiental, 8(11), 1-9. Recuperado de https://tinyurl.com/y48xapzo

Montaño, L. \& Solís, P.C. (2001). Modernidad e institucionalidad universitarias. Desafíos y transformaciones socio-organizacionales. Denarius Revista de Economía y Administración, 2(3), 13-32. Recuperado de https://tinyurl.com/yxzhduwb

Naciones Unidas. (1972). Declaración de Estocolmo sobre el medio ambiente humano. Recuperado de https:// tinyurl.com/nwzfgkm

Nieto, L.M. \& Medellín, P. (2007). Medio ambiente y educación superior: implicaciones en las políticas públi- cas. Revista de la Educación Superior, 142 , 31-42. Recuperado de https://tinyurl.com/y34uwlp6

Novo, M. (2009). La educación ambiental, una genuina educación para el desarrollo sostenible. Revista de Educación, (No. Extraordinario), 195-217. Recuperado de https://tinyurl.com/y53yyj9j

Orr, D.W. (2004). Earth in Mind: On Education, Environment, and the Human Prospect. Washington: Island Press. Recuperado de https://tinyurl.com/y5dduawz

Pérez, A., Julián, J.A. \& López, V.M. (2009). V.M. López Pas tor (Coord.). Evaluación formativa y compartida en Educación Superior. Propuestas, técnicas, instru mentos y experiencias. (pp. 19-44). Madrid: Narcea Recuperado de https://tinyurl.com/y3ztwe5k

Ramírez, R. (2008). La pedagogía crítica. Una manera ética de generar procesos educativos. Folios, 28, 108-119. https://doi.org/10.17227/01234870.28folios108.119

Retamoso, G. (2007). Educación y Sociedad. Civilizar. Ciencias Sociales y Humanas, 7(12), 171-186. https://doi. org/10.22518/16578953.789

Santos, M.A. (2011). Sostenibilidad y educación intercultural. El cambio de perspectiva. Bordón, 63(4), 123 135. Recuperado de https://tinyurl.com/yykyc2d8

Southwell, M. (2013). La escuela ante nuevos desafíos: participación, ciudadanía y nuevas alfabetizaciones. Buenos Aires: Fundación Santillana. Recuperado de https://tinyurl.com/y3yvvhfn

Torres, R. (2012). Educación pública universitaria. Visión estratégica en favor del desarrollo. La Plata: Dei Genitrix. Recuperado de https://tinyurl.com/y46olose Villavicencio, S. (2008). Filosofías de la universidad. Fines y responsabilidades de la universidad en el nuevo marco mundial. En: G. Hoyos Vásquez (Ed.) Filosofía de la educación. (pp. 321-340). Madrid: Editorial Trotta y Consejo Superior de Investigaciones Científicas. Recuperado de https://tinyurl.com/yxdwxx2a

RIDU / Revista Digital de Investigación en Docencia Universitaria / e-ISSN: 2223-2516

(C) Los autores. Este artículo es publicado por la Revista Digital de Investigación en Docencia Universitaria del Área de Institutional Research and Effectiveness de la Dirección de Aseguramiento de la Calidad, Universidad Peruana de Ciencias Aplicadas. Este es un artículo de acceso abierto, distribuido bajo los términos de la LicenciaCreativeCommons Atribución-CompartirIgual 4.0 Internacional.(http://creativecommons.org/licenses/by-sa/4.0/), que permite el uso no comercial, distribución y reproducción en cualquier medio, siempre que la obra original sea debidamente citada. 